\title{
Moderate sedation helps improve future behavior in pediatric dental patients - a prospective study
}

\section{Denise Espíndola ANTUNES(a) Karolline Alves VIANA ${ }^{(b)}$ Paulo Sucasas COSTA ${ }^{(c)}$ Luciane Rezende COSTA(d)}

(a) Universidade Federal de Goiás - UFG, Health Sciences Graduate Program, Goiânia, Goiás, Brazil.

(b) Universidade Federal de Goiás - UFG, Dentistry Graduate Program, Goiânia, Goiás, Brazil.

(c) Universidade Federal de Goiás - UFG, School of Medicine, Department of Pediatrics, Goiânia, Goiás, Brazil.

(d) Universidade Federal de Goiás - UFG, School of Dentistry, Division of Pediatric Dentistry, Goiânia, Goiás, Brazil.

Declaration of Interests: The authors certify that they have no commercial or associative interest that represents a conflict of interest in connection with the manuscript.

\section{Corresponding Author:}

Luciane Rezende Costa

E-mail: Isucasas@ufg.br

DOI: 10.1590/1807-3107BOR-2016.vol30.0107

Submitted: Nov 21, 2015

Accepted for publication: May 11, 2016

Last revision: Jul 20, 2016
Abstract: There is little evidence on the long-term effects of pharmacological management in children undergoing dental treatment. This study aimed to assess children's behavior in consecutive dental sessions following oral rehabilitation using different pharmacological regimens for behavioral control. Participants were preschoolers who were previously treated for caries under one of the following: no sedative, oral sedation with midazolam, oral sedation with midazolam/ketamine, or general anesthesia. The children's behavior in the follow-up sessions was assessed using the Ohio State University Behavioral Rating Scale (OSUBRS); higher scores represented less cooperative behavior (range 5-20). Follow-up assessments were conducted on 50 children under four years old for up to 29 months. Data were analyzed by the Friedman/Wilcoxon tests and Cox regression model. OSUBRS mean (standard deviation) scores for the whole sample decreased from 11.9 (5.4) before treatment to 6.8 (3.2) at the final recall session $(\mathrm{p}<0.001)$. Moderate sedation with midazolam (OR 2.9, 95\%CI 1.2-6.9) or midazolam/ketamine (OR 4.3, 95\%CI 1.6-11.4) improved children's future behavior. The general anesthesia group ( $n=4)$ had a small sample size and the results should be considered with caution. Although invasive dental treatment negatively affected the child's behavior in the dental chair, they became more cooperative over time. Moderately sedated children showed better prospective behavior than those in the non-sedation group.

Keywords: Dental Caries; Restraint, Physical; Conscious Sedation; Anesthesia, General; Dental Care for Children.

\section{Introduction}

At present there is an extensive debate over the best method of behavioral management of preschool-aged children undergoing dental treatment ${ }^{1,2,3,4,5}$. Protective stabilization, moderate sedation, and general anesthesia are advanced behavioral control techniques indicated during the dental treatment of early childhood caries. These methods offer advantages and disadvantages during and immediately after the procedure ${ }^{6}$. However, retrospective studies have shown conflicting results with regard to the long-term behavior of children during dental treatment, with some studies reporting better long-term acceptance of dental treatment by children with the tell-show-do method compared with moderate sedation or 
general anesthesia7. Others have reported equally improved behavior among children who underwent restraint or general anesthesia at short-term recall visits $^{8}$, as well as no differences between general anesthesia and sedation'. Moreover, no differences were observed between non-pharmacological control and sedation ${ }^{10}$, and better results were obtained with general anesthesia compared with sedation ${ }^{11}$.

Many children require invasive dental treatment due to early childhood caries. According to the final report of a large epidemiological survey on the oral condition of Brazilians ${ }^{12}$, children aged 5 years had an average of 2.4 primary teeth with caries, and fewer than $20 \%$ of these had been treated in 2010. This disease continues to remain a major public health problem in many countries, with 9\% (621 million people worldwide) of the global population $^{13}$ and $19.5 \%$ of American children ${ }^{14}$ between ages 2 and 5 years affected by untreated caries in the primary teeth in 2010.

Dentists often wonder how to provide maximum benefit during pediatric dental treatment while simultaneously minimizing the risk of an unpleasant dental experience that may traumatize the child. Adults with dental phobia report suffering from dental treatment in their childhood ${ }^{15,16,17}$, and data from different countries show that the prevalence of dental anxiety in adulthood varies between $13.5 \%{ }^{17}$ and $36.9 \%{ }^{18}$. Likewise, approximately $20 \%$ of Brazilian patients exhibit moderate to severe anxiety associated with dental treatment ${ }^{19}$.

Therefore, the aim of this prospective study was to compare the long-term behavior of children who underwent advanced behavioral techniques (nonpharmacological control, moderate sedation, or general anesthesia) while receiving treatment for dental caries. Our hypothesis was that pharmacological approaches would facilitate children's cooperation with the dentist in follow-up sessions by potentially diminishing their distress during dental treatment.

\section{Methodology}

\section{Study design}

This prospective study stems from a randomized clinical trial (NCT00902395) that compared patient behavior among four different groups during follow-up dental visits after completion of invasive dental procedures in children under four years of age. Children were treated for early childhood caries and were assigned to different treatment groups: no sedative, oral sedation with midazolam, oral sedation with midazolam/ketamine, or general anesthesia. This study was approved by the Institutional Review Board (IRB) of the Universidade Federal de Goiás (Protocol number: 219/09) and followed the ethical principles of the Declaration of Helsinki. Informed consent was obtained from parents after a thorough discussion about the objectives, procedures, risks, and benefits of the study.

\section{Participants}

Fifty-six children under four years old who completed the trial were recruited for the follow-up phase. They were initially included in the trial if they were cleared by pediatric professionals to receive moderate sedation or general anesthesia. That is, patients that were healthy (American Society of Anesthesiologists, Physical Status I), demonstrated negative behavior in previous dental appointments, and were in need of extensive dental treatment ${ }^{6}$. Children were also included if they had no physical or mental disabilities and had never had any dental treatment. They were excluded from the follow-up phase if their parents refused to attend the periodic examinations, but data from children who attended the dental sessions for at least one year were kept for analysis.

The sample size for this study was calculated based on a previous study ${ }^{7}$ that reported that $78 \%$ of 146 children aged 3-13 years showed good cooperation five years after dental treatment, $18 \%$ required moderate sedation, and $4 \%$ underwent general anesthesia. A minimum sample size of 10 children per group was needed to obtain $5 \%$ and $80 \%$ power for a two-sided hypothesis test.

\section{Proceedings of the randomized clinical trial phase}

Six children participated in a pilot study to test the trial protocol; no changes were needed. Although the entire trial protocol has been described previously ${ }^{1}$, 
it is important to emphasize some aspects of the study to improve understanding.

During the recruitment stage, a pediatrician and an anesthesiologist assessed the children's general health and a pediatric dentistry assessed their oral health. Children were definitely included in the trial if the physicians and the dentist agreed upon the need for sedation.

Children were randomly assigned to the intervention groups by shuffling opaque sealed envelopes. One member of the research team shuffled the envelopes, pulled one out, and allocated the children to one of four study groups: no sedation (control group) $(\mathrm{n}=17)$; moderate sedation with midazolam (Dormire ${ }^{\circledR}$, Cristália, São Paulo, Brazil; dose $1 \mathrm{mg} / \mathrm{kg}$, maximum $20 \mathrm{mg}$ ) administered orally $(\mathrm{n}=16)$; moderate sedation with the association of midazolam $(0.5 \mathrm{mg} / \mathrm{kg}$, maximum $20 \mathrm{mg})$ and ketamine (S+ Ketamin ${ }^{\circledR}$, Cristália, São Paulo, Brazil, $3 \mathrm{mg} / \mathrm{kg}$ dose, maximum $50 \mathrm{mg}$ ) administered orally ( $n=13)$; and general anesthesia with sevoflurane, propofol, fentanyl, and premedication with midazolam $(n=4)$. All children, except the general anesthesia group, were treated under protective stabilization where the child was wrapped in a sheet fixed with tape.

After randomization, if a parent did not agree with the intervention, the child was excluded from the study. Also, parents were aware that they could forgo their children's participation in the study at any time. In Brazil, protective stabilization without sedation is more common than pharmacological techniques for behavior management in children undergoing dental treatment. Therefore, the IRB did not identify any ethical issues with the randomization process or the performance of extensive dental treatment under protective stabilization.

The children were aged under 4 years when participating in the trial study, and had a mean decayed, missing, and filled teeth index of 6.9 (standard deviation 'SD' 4.9) (Table 1). One of three certified pediatric dentists performed the surgical-restorative dental treatments for each child in as many sessions as were needed $(6.2, \mathrm{SD} 4.3)$, except in the case of general anesthesia, in which the whole treatment was completed in one extended session (Table 1). The sessions without medication and with sedation were carried out at an outpatient level, and the children were accompanied by an adult throughout the session. The general anesthesia sessions were carried out in a day-hospital type surgical center. Children received comparable invasive dental procedures (Table 1). Local anesthetic ( $2 \%$ lidocaine $+1: 100000$ epinephrine, maximum $4.4 \mathrm{mg} / \mathrm{kg}$ ) was administered to all groups in all sessions.

One of the two anesthesiologists were responsible for administering the sedatives and general anesthesia, as well as for monitoring the patients. During sedation, each child's oxygen saturation and heart rate were monitored by the 3800 pulse oximeter (Datex Ohmeda Inc., Madison, Wisconsin), and during general anesthesia the children received additional electrocardiography and capnography monitoring.

\section{Follow-up procedures}

The follow-up sessions (or recall appointments) commenced approximately 4 months after completion of the dental treatment and were performed at intervals of approximately 4 months, depending on the participant's attendance. The total follow-up was planned to be completed in 24 months. The mean age of the children at the beginning and end of follow-up were 34.0 (SD 6.5) and 52.1 (5.7) months old, respectively.

At each follow-up session, the children underwent a standardized dental check-up that was identical to the initial dental visit (trial phase) where the baseline behavior of the child was determined and a treatment plan established. One of four certified pediatric dentists examined the child without the use of protective stabilization and in the presence of an accompanying adult. All pediatric dentists were trained to use distraction and tell-show-do techniques during the dental examination. The dentist first performed a professional prophylaxis with a rubber cup, followed by a dental intraoral examination using a dental mirror and an exploratory probe under adequate lighting. The child was discharged after a topical application of fluoride varnish.

The child's behavior at baseline (before dental treatment) and follow-up sessions was recorded by the same dentist (observer), who was different from the pediatric dentists in charge of the dental exams. The 
Table 1. Children's demographic information and dental sessions' characteristics by the experimental group.

\begin{tabular}{|c|c|c|c|c|c|}
\hline \multirow[b]{2}{*}{ Characteristics } & \multicolumn{4}{|c|}{ Experimental group $-\mathrm{n}$ or mean (standard deviation) } & \multirow{2}{*}{$\begin{array}{c}\text { Total } \\
\text { sample }\end{array}$} \\
\hline & $\begin{array}{c}\text { No } \\
\text { sedative }\end{array}$ & $\begin{array}{l}\text { Midazolam } \\
\text { sedation }\end{array}$ & Midazolam/ketamine sedation & $\begin{array}{c}\text { General } \\
\text { anesthesia }\end{array}$ & \\
\hline \multicolumn{6}{|l|}{ Baseline session (before the dental treatment) } \\
\hline $\mathrm{n}$ & 17 & 16 & 13 & 4 & \\
\hline Child's age, months & $28.1(6.0)$ & $25.4(5.7)$ & $28.9(6.1)$ & $29.3(9.7)$ & $27.5(6.2)$ \\
\hline Male & 10 & 11 & 7 & 1 & 29 \\
\hline Dental exam length, minutes & $12.9(4.0)$ & $10.6(1.7)$ & $11.5(3.2)$ & $11.3(2.5)$ & $11.7(3.1)$ \\
\hline dmft & $5.8(3.6)$ & $6.8(5.2)$ & $5.6(3.5)$ & $16.3(6.8)$ & $6.9(4.9)$ \\
\hline OSUBRS, sum* & $12.5(5.3)$ & $13.8(4.7)$ & $11.3(5.2)$ & $9.5(4.1)$ & $12.4(5.0)$ \\
\hline \multicolumn{6}{|l|}{ Dental treatment } \\
\hline Appointments needed for completion of treatment & $3.0(1.7)$ & $3.0(1.5)$ & $2.7(2.0)$ & 1.0 & $3.2(1.7)$ \\
\hline \multicolumn{6}{|l|}{ Procedures } \\
\hline Restoration only & 14 & 10 & 9 & 1 & 34 \\
\hline Pulp therapy and restoration & 1 & 2 & 2 & & 5 \\
\hline Exodontia and restoration & 1 & 3 & 1 & 3 & 8 \\
\hline Exodontia, pulp therapy and restoration & 1 & 1 & 1 & & 3 \\
\hline \multicolumn{6}{|l|}{ Follow-up 1} \\
\hline $\mathrm{n}$ & 17 & 16 & 13 & 4 & 50 \\
\hline Time elapsed from baseline, months & $5.8(1.8)$ & $6.8(2.1)$ & $6.4(2.3)$ & $8.5(2.6)$ & $6.5(2.2)$ \\
\hline Child's age, months & $33.8(5.6)$ & $32.3(6.3)$ & $35.2(6.4)$ & $37.8(10.4)$ & $34.0(6.5)$ \\
\hline Dental exam length, minutes & $10.2(2.2)$ & 10.0(1.8) & $11.9(2.5)$ & $12.5(2.9)$ & $10.8(2.4)$ \\
\hline OSUBRS, sum* & $10.8(4.0)$ & $8.6(4.7)$ & $8.4(4.7)$ & $12.0(5.9)$ & $9.6(4.6)$ \\
\hline \multicolumn{6}{|l|}{ Follow-up 2} \\
\hline $\mathrm{n}$ & 17 & 16 & 13 & 4 & 50 \\
\hline Time elapsed from baseline, months & $9.5(2.1)$ & $10.3(2.1)$ & $10.5(2.5)$ & $12.8(7.7)$ & $10.3(2.4)$ \\
\hline Child's age, months & $37.5(6.1)$ & $35.6(6.2)$ & $39.3(6.0)$ & $42.0(10.7)$ & $37.7(6.6)$ \\
\hline Dental exam length, minutes & $12.7(5.3)$ & $11.9(4.0)$ & $10.8(1.9)$ & $12.5(8.3)$ & $11.9(4.0)$ \\
\hline OSUBRS, sum* & $10.2(5.0)$ & $8.1(4.4)$ & $7.7(4.4)$ & $6.8(2.2)$ & $8.6(4.6)$ \\
\hline \multicolumn{6}{|l|}{ Follow-up 3} \\
\hline $\mathrm{n}$ & 17 & 16 & 13 & 4 & 50 \\
\hline Time elapsed from baseline, months & $12.1(2.4)$ & $14.0(2.4)$ & $14.4(2.9)$ & $16.5(9.7)$ & $14.1(2.7)$ \\
\hline Child's age, months & $41.4(5.9)$ & $39.4(6.3)$ & $43.2(5.9)$ & $45.8(11.1)$ & $41.6(6.6)$ \\
\hline Dental exam length, minutes & $10.6(1.7)$ & $10.9(2.0)$ & $10.8(2.8)$ & $11.3(2.5)$ & $10.8(2.1)$ \\
\hline OSUBRS, sum* & $9.7(4.4)$ & $7.7(4.3)$ & $5.9(2.5)$ & $6.3(2.5)$ & $7.8(4.1)$ \\
\hline \multicolumn{6}{|l|}{ Follow-up 4} \\
\hline $\mathrm{n}$ & 13 & 13 & 4 & 0 & 30 \\
\hline Time elapsed from baseline, months & $17.1(1.2)$ & $18.3(2.1)$ & $15.5(2.4)$ & - & $17.4(2.0)$ \\
\hline Child's age, months & $44.1(5.7)$ & $44.6(5.3)$ & $45.0(2.4)$ & - & $44.4(5.1)$ \\
\hline Dental exam length, minutes & $9.6(3.8)$ & $9.6(3.2)$ & $12.5(2.9)$ & - & $10.0(3.5)$ \\
\hline OSUBRS, sum* & $8.1(2.9)$ & $5.5(1.0)$ & $5.0(0)$ & - & $6.5(2.4)$ \\
\hline \multicolumn{6}{|l|}{ Follow-up 5} \\
\hline $\mathrm{n}$ & 13 & 13 & 4 & 0 & 30 \\
\hline Time elapsed from baseline, months & $21.2(2.0)$ & $22.7(2.8)$ & $19.0(2.4)$ & - & $21.5(2.7)$ \\
\hline Child's age, months & $48.1(5.7)$ & $49.0(5.7)$ & $48.3(2.2)$ & - & $48.5(5.3)$ \\
\hline Dental exam length, minutes & $11.2(2.2)$ & 10.5(3.0) & $11.3(2.5)$ & - & $10.9(2.5)$ \\
\hline OSUBRS, sum* & $7.8(2.6)$ & $5.2(0.4)$ & $5.0(0)$ & - & $6.3(2.2)$ \\
\hline \multicolumn{6}{|l|}{ Follow-up 6} \\
\hline $\mathrm{n}$ & 12 & 12 & 4 & - & 28 \\
\hline Time elapsed from baseline, months & $25.3(2.0)$ & $26.3(1.7)$ & $22.3(3.8)$ & - & $25.3(2.5)$ \\
\hline Child's age, months & $52.7(5.8)$ & $52.7(5.8)$ & $48.8(5.3)$ & - & $52.1(5.7)$ \\
\hline Dental exam length, minutes & $11.7(3.6)$ & $12.5(3.4)$ & $12.5(2.9)$ & - & $12.1(3.2)$ \\
\hline OSUBRS, sum* & $8.3(4.0)$ & $5.8(2.1)$ & $5.0(0)$ & - & $6.8(3.2)$ \\
\hline
\end{tabular}

$\mathrm{dmft}$ : decayed, missing, filled teeth; *Sum of the highest scores observed in five periods related to key procedures, using the Ohio State University Behavioral Rating Scale (OSUBRS). Sums ranged between 5 and 20; the higher the OSUBRS sum, the worse the child's behavior 
observer was an experienced dentist trained in the proper use of the scale based on 15 minute-extracts of videos recorded during the children's dental treatments. Simultaneously, the observer also scored the children's behavior every minute according to the Ohio State University Behavioral Rating Scale (OSUBRS) ${ }^{20}$ through observation of the movements of the head and extremities, crying, and physical resistance. The observer scored the same video files again one week later, and the intra-rater agreement was found to be satisfactory (weighted Kappa 0.8). The OSUBRS is comprised of four mutually exclusive scores: 1) behavior without crying and without movements (quiet behavior); 2) behavior with crying and no movements; 3 ) behavior with movements without crying; 4) behavior with crying and movements (resistance behavior).

For data collection, the OSUBRS method was adapted to facilitate in situ observation instead of recording a videotape. The observer was unmasked, took part in the entire dental consultation, and scored the child's behavior according to the highest OSUBRS score detected during the periods related to key procedures, including when the child entered the clinic, the professional prophylaxis, the intraoral examination, topical fluoride varnish application, and at the end of the dental procedure. The sum of the OSUBRS scores registered in each of the 5 periods in a session was calculated to determine a total score/session (minimum 5, maximum 20).

\section{Statistical analyses}

Data were tabulated using IBM SPSS version 19.0 software and quality-checked. Frequencies of OSUBRS scores and measures of dispersion were calculated. The Friedman's and Wilcoxon tests were used to compare the changes in the sum of OSUBRS scores observed in each follow-up session. Significance was set at $\mathrm{p}<0.05$.

Survival analysis was chosen to analyze the length of time required for children to show cooperative behavior in a routine recall appointment (follow-up session as described earlier) as a function of predictor variables. Survival analysis was chosen instead of regression or ANOVA because it allows censored values such as cases lost to follow-up. The event of interest was the first time a child reached "quiet" behavior (OSUBRS score $=1$ ) at $100 \%$ of the five evaluation points during follow-up sessions (sum of OSUBRS scores $=5$ ). The response variable was the amount of time that elapsed between the baseline consultation and the end of follow-up sessions or the censorship of cases. The censored cases were those that had abandoned follow-up visits or exhibited resistance behaviors (crying and/or movement).

Cox's proportional hazard model evaluated explanatory variables for quiet behavior including gender, age, and type of behavioral intervention. This model is a multiple regression analysis applied to survival analysis. Using Cox's model an estimate of the role of independent variables that act multiplicatively on risk is obtained. The assumption was that different individuals have certain traits that are proportional to each other and that the ratio among these risk functions does not vary over time.

\section{Results}

The parents of 50 children provided consent, while the parents of 6 children refused to participate in the follow-up phase. Children were followed-up for up to 29 months, with a mean follow-up time of 25.3 months (SD 2.5). None of the children included in the study required additional operative care in the period between completion of treatment and the final follow-up session. Details of each consultation and the demographic characteristics are shown in Table 1. The Friedman test showed statistically significant differences between the sums of OSUBRS scores in different consultations $(\mathrm{p}<0.001)$. Post-hoc comparisons (Wilcoxon) showed that children's behavior improved over time, with it being more negative at baseline than at the first follow-up $(p=0.001)$, exhibiting no significant changes from follow-up sessions 1-3 ( $p>0.05)$, enhanced changes at follow-up $4(p=0.019)$, and no further changes of significance through the end of the study ( $p>0.05)$.

The frequencies of the different OSUBRS scores are shown in Figure 1. The five time points for each session (entering the office, prophylaxis, examination, fluoride application, and the end of the consultation) were investigated separately. The percentage of "quiet" scores tended to increase over time, from $40.0 \%$ at 
baseline to a peak of $77.3 \%$ in the fifth session. At the end of the follow-up period, $76.4 \%$ of children exhibited 'quiet' behavior at all five time points. Once a child reached a quiet behavior during the whole session, $\mathrm{s} /$ he kept this behavior in the on-going sessions.

Survival analysis of the 'gender', 'age at baseline', and 'intervention' variables showed that the children most likely to display totally quiet behavior during follow-up were those who received sedation with

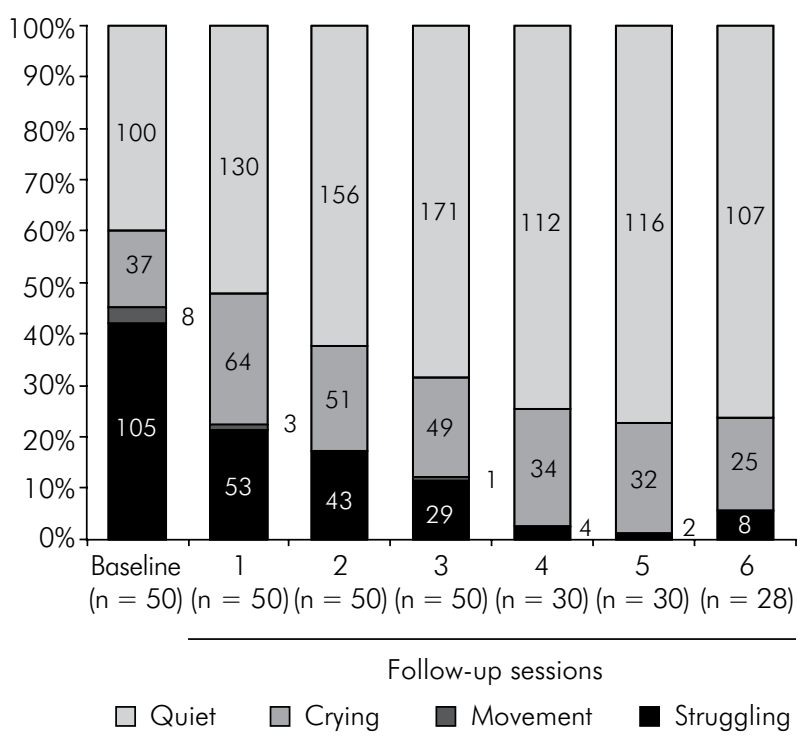

*OSUBRS: Ohio State University Behavioral Rating Scale.

Figure 1. Relative and absolute frequencies of OSUBRS* scores obtained during the different follow-up sessions representing the highest behavioral score observed at 5 key intervals.

Table 2. Cox regression model for independent variables explaining quiet behavior (OSUBRS score $=1$; scores assessed at 5 intervals during follow-up dental exams).

\begin{tabular}{lcc}
\hline $\begin{array}{l}\text { Independent variable } \\
\text { Gender }\end{array}$ & $\begin{array}{c}\text { Odds Ratio (95\% } \\
\text { Confidence Interval) }\end{array}$ & $p$ \\
$\quad \begin{array}{l}\text { Female } \\
\text { Male }\end{array}$ & 1.0 & 0.705 \\
Age at baseline (months) & $1.1(0.6-2.3)$ & 0.221 \\
$\begin{array}{l}\text { Intervention } \\
\text { No sedative }\end{array}$ & $1.0-1.1)$ & \\
Midazolam & $2.9(1.2-6.9)$ & 0.017 \\
Midazolam+ketamine & $4.3(1.6-11.4)$ & 0.004 \\
General anesthesia & $1.8(0.4-7.0)$ & 0.427 \\
\hline
\end{tabular}

Overall chi-square 11.469, $\mathrm{p}=0.043$; OSUBRS: Ohio State University Behavioral Rating Scale. midazolam (2.9 times more likely) and those who received midazolam + ketamine (4.3 times as likely). Other variables showed no significant results (Table 2). The survival table (Figure 2) showed that $50 \%$ of children in the midazolam-ketamine group displayed 'quiet behavior' 10 months after the baseline consultation, and this percentage was lower for the other groups (approximately 37\% for midazolam, $25 \%$ for general anesthesia, and $17 \%$ for the group that received no medication).

\section{Discussion}

Our results demonstrate that children who received moderate sedation during surgical-restorative dental treatment for early childhood caries behaved more positively during follow-up sessions than those patients who did not receive sedation. In general, child behavior in the dental office improves along with increasing psychomotor development ${ }^{21,22}$. This investigation provides scientific evidence that the use of sedatives can diminish the negative impact of invasive dental treatments compared with the exclusive use of protective stabilization (without sedative).

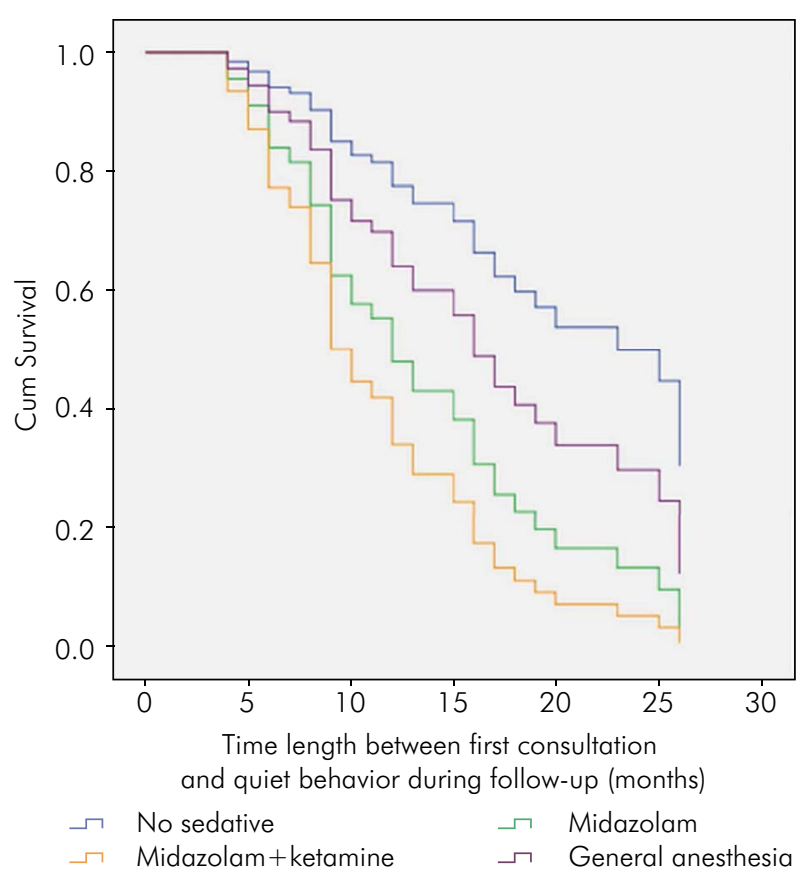

Figure 2. Life table for quiet behavior during the follow-up session after dental treatment rendered according to one of the intervention groups. 
We anticipated the use of midazolam would lead to improved behavior during follow-up sessions due to the ability of this sedative to promote anterograde amnesia. Midazolam-induced amnesia could have led to better medium-term behavior in this study because the children likely did not remember the dental procedure. One study has shown that midazolam produces amnesia ten minutes after oral administration ${ }^{23}$. Another pediatric dentistry-based study showed that $80 \%$ of children under 72 months of age were unable to recall figures shown after administration of midazolam ${ }^{24}$.

In this study, the group that received ketamine and midazolam exhibited better behaviors during follow-up sessions than the others. Like midazolam, ketamine has the ability to promote anterograde amnesia ${ }^{25}$. In addition to amnesia, ketamine is expected to reduce pain perception and improve a child's comfort level during invasive procedures ${ }^{26,27}$.

The long-term results from the addition of ketamine as a sedative can be explained by the phenomenon of "implicit memory". Midazolam affects patient memory as far as perceptual recognition and facilitation are concerned $^{28}$. That is, children sedated with a moderate level of midazolam remain responsive to verbal and physical stimuli but do not recognize images seen after administration of the sedative (explicit memory). Meanwhile, patients continue to store information on what is happening during sedation (implicit memory), which has a direct effect on subsequent behavior. Therefore, with the addition of ketamine, we hypothesize that the better behavior of the group receiving this both ketamine and midazolam can be explained by the reduced perception of pain. Thus, those children would have an implicit memory of greater comfort during dental treatment.

The group of children who received no sedative were less cooperative over time compared to the groups who received at least some sedation. Crying and movement in these children indicated the no-sedative group considered dental treatment to be unpleasant. However, another study ${ }^{10} \mathrm{did}$ not document any differences in the behavior of children 2 to 34 months following dental treatment where children were sedated; the study compared different sedation methods: midazolam and nitrous oxide, chloral hydrate and nitrous oxide, and no pharmacological agents. Their study ${ }^{10}$, however, was retrospective and not controlled.

The results for the general anesthesia group should be viewed with caution due to the small sample size. One prospective 5-year follow-up study found that more than one-half of children between 0 and 13 years old that had received general anesthesia showed a total lack of cooperation and/or fear during subsequent dental visits. ${ }^{29}$ Retrospective studies of children treated under general anesthesia and mild sedation, not including midazolam, found no differences in future behaviors 13 months and 18 months, respectively, after completion of dental treatment ${ }^{11,30}$. In our study, the results of the general anesthesia group did not differ from those of the non-medicated group. Additional studies to evaluate the future behavior of children who receive general anesthesia as compared to those sedated with midazolam are needed.

The sums of OSUBRS scores significantly decreased over time. Studies with different protocols indicate that child behavior during dental visits generally improves with psychomotor development ${ }^{21,22}$. This improvement is facilitated if children have not experienced painful or unpleasant procedures or by having recurring visits to the dentist. ${ }^{22}$ Patient age at the onset of dental care also influences child behaviour ${ }^{31}$. Nonetheless, our results indicate age at baseline visit did not influence patient behavior. This is probably because sedatives exert a greater influence on behavior than age during treatment.

It should be emphasized that, regardless of the benefits demonstrated in this study, sedation should not be used indiscriminately. The dentist, i.e. certified pediatric dentist, should have specific knowledge and training to be responsible for sedation of children during dental treatment ${ }^{32}$. In Brazil, training in sedation is rarely addressed in undergraduate and graduate programs. Furthermore, some drugs cannot be used without the supervision of an anesthesiologist; ketamine, for example, is categorized as a general anesthetic ${ }^{1}$.

Despite our efforts to control for variables in this study, there were some limitations with this study. The investigator, pediatric dentists, anesthesiologists, and the caretakers of patients were aware of the type of intervention each child received. Intervention 
masking was not proposed because general anesthesia was initially included in the experimental study. In addition, while a follow-up rate of $100 \%$ within the first year, the final follow-up rate was $56.0 \%$.

As the survival analysis showed a remarkable difference in child's behavior around the tenth month of follow-up, the results of the sedation groups compared to no sedation might be generalized to other patient groups. However, due to small sample size, the results of the general anesthesia group should not be generalized ${ }^{33}$.

\section{Conclusion}

Overall, this study contributes to the field of child behavior management. The dental treatment of early childhood caries under moderate sedation was shown to significantly improve the future behavior of children during subsequent recall appointments 4 to 29 months after completion of treatment. Moderate sedation can be a valuable tool in reducing the suffering of toddlers and preschoolers during dental treatment. Nonetheless, pediatric dentists must receive special training to administer sedation.

\section{Acknowledgements}

We would like to thank Anelise Daher, Cristiana M. Jesus-França, Julianna A. Cavalcante, Sarah V. Brasileiro, Hugo Sérgio G. Oliveira, and Thiago A. C. Moreira for their support throughout this study.

\section{References}

1. Moreira TA, Costa PS, Costa LR, Jesus-França $\mathrm{CM}$, Antunes DE, Gomes HS et al. Combined oral midazolam-ketamine better than midazolam alone for sedation of young children: a randomized controlled trial. Int J Paediatr Dent. 2013;23(3):207-15. doi:10.1111/j.1365-263X.2012.01246.x

2. Hand D, Averley P, Lyne J, Girdler N. Advanced paediatric conscious sedation: an alternative to dental general anaesthetic in the U.K. SAAD Dig. 2011;27:24-9. doi:

3. Berg M. Dental fear in children: clinical consequences Suggested behaviour management strategies in treating children with dental fear. Eur Arch Paediatr Dent. 2008;9 Suppl 1:41-6. doi:10.1007/BF03262655

4. Wilson S. Management of child patient behavior: quality of care, fear and anxiety, and the child patient. Pediatr Dent. 2013;35(2):170-4. doi:10.1016/j.joen.2012.11.040

5. Lourenço-Matharu L, Ashley PF, Furness S. Sedation of children undergoing dental treatment. Cochrane Database Syst Rev. 2012;(3):CD003877. doi:10.1002/14651858.CD003877.pub4

6. American Academy on Pediatric Dentistry Clinical Affairs Committee-Behavior Management Subcommittee; American Academy on Pediatric Dentistry Council on Clinical Affairs. Guideline on behavior guidance for the pediatric dental patient. Pediatr Dent. 2008-2009;30(7 Suppl):125-33.

7. Varpio M, Wellfelt B. Some characteristics of children with dental behaviour problems. Five-year follow-up of pedodontic treatment. Swed Dent J. 1991;15(2):85-93.

8. Zhang HM, Xia B, Wang JH, Chen XX, Ge LH. [Influence of the effect of general anaesthesia and restraint during dental treatment on dental anxiety and behavior in children]. Beijing Da Xue Xue Bao. 2015;47(1):134-9. Chinese.

9. Kupietzky A, Blumenstyk A. Comparing the behavior of children treated using general anesthesia with those treated using conscious sedation. ASDC J Dent Child. 1998;65(2):122-7.

10. McComb M, Koenigsberg SR, Broder HL, Houpt M. The effects of oral conscious sedation on future behavior and anxiety in pediatric dental patients. Pediatr Dent. 2002;24(3):207-11.

11. Fuhrer CT 3rd, Weddell JA, Sanders BJ, Jones JE, Dean JA, Tomlin A. Effect on behavior of dental treatment rendered under conscious sedation and general anesthesia in pediatric patients. Pediatr Dent. 2009;31(7):492-7.

12. Ministério da Saúde (BR). Divisão Nacional de Saúde Bucal. SBBrasil 2010: Pesquisa Nacional de Saúde Bucal: Resultados principais. Brasília, DF: Ministério da Saúde; 2011[cited 2015 Jul 28]. Available from: http://www.webcitation.org/69Q2z4bI2

13. Kassebaum NJ, Bernabé E, Dahiya M, Bhandari B, Murray CJ, Marcenes W. Global burden of untreated caries: a systematic review and metaregression. J Dent Res. 2015;94(5):650-8. doi:10.1177/0022034515573272

14. National Center for Health Statistics (USA). Health, United States, 2009 with special feature on medical technology. Hyattsville: National Center for Health Statistics; 2010. (Report, vol 2010-1232).

15. Oliveira MA, Bendo CB, Ferreira MC, Paiva SM, Vale MP, Serra-Negra JM. Association between childhood dental experiences and dental fear among dental, psychology and mathematics undergraduates in Brazil. Int J Environ Res Public Health. 2012;9(12):4676-87. doi:10.3390/ijerph9124676

16. Humphris G, King K. The prevalence of dental anxiety across previous distressing experiences. J Anxiety Disord. 2011;25(2):232-6. doi:10.1016/j.janxdis.2010.09.007 
17. Nicolas E, Collado V, Faulks D, Bullier B, Hennequin M. A national cross-sectional survey of dental anxiety in the French adult population. BMC Oral Health. 2007;7(1):12. doi:10.1186/1472-6831-7-12

18. Pohjola V, Mattila AK, Joukamaa M, Lahti S. Anxiety and depressive disorders and dental fear among adults in Finland. Eur J Oral Sci. 2011;119(1):55-60. doi:10.1111/j.1600-0722.2010.00795.x

19. Carvalho RWF, Falcão PGCB, Campos GJL, Bastos AS, Pereira JC, Pereira MA et al. [Anxiety regarding dental treatment: prevalence and predictors among Brazilians]. Ciênc Saúde Coletiva. 2012;17(7):1915-22. Portuguese. doi:10.1590/S1413-81232012000700031

20. Torres-Pérez J, Tapia-Garcĺa I, Rosales-Berber MA, Hernández-Sierra JF, Pozos-Guillén AJ. Comparison of three conscious sedation regimens for pediatric dental patients. J Clin Pediatr Dent. 2007; 31(3):183-6. doi:10.17796/jcpd.31.3.e82526q0432375n0

21. Rayen R, Muthu MS, Chandrasekhar Rao R, Sivakumar $\mathrm{N}$. Evaluation of physiological and behavioral measures in relation to dental anxiety during sequential dental visits in children. Indian J Dent Res. 2006;17(1):27-34. doi:10.4103/0970-9290.29895

22. Cunha RF, Zaze ACSF, Vieira AEMY, Melhado FL, Sundefeld ML. Longitudinal behavioral analysis during dental care of children aged 0 to 3 years. Braz Oral Res. 2009;23(3):302-6. doi:10.1590/S1806-83242009000300013

23. Kain ZN, Hofstadter MB, Mayes LC, Krivutza DM, Alexander G, Wang SM et al. Midazolam: effects on amnesia and anxiety in children. Anesthesiology. 2000;93(3):676-84. doi:10.1097/00000542-200009000-00016

24. Al-Zahrani AM, Wyne AH, Sheta SA. Comparison of oral midazolam with a combination of oral midazolam and nitrous oxide-oxygen inhalation in the effectiveness of dental sedation for young children. J Indian Soc Pedod Prev Dent. 2009;27(1):9-16. doi:10.4103/0970-4388.50810
25. Singh C, Pandey RK, Saksena AK, Chandra G. A comparative evaluation of analgo-sedative effects of oral dexmedetomidine and ketamine: a triple-blind, randomized study. Paediatr Anaesth. 2014;24(12):1252-9. doi:10.1111/pan.12493

26. Bui T, Redden RJ, Murphy S. A comparison study between ketamine and ketamine-promethazine combination for oral sedation in pediatric dental patients. Anesth Prog. 2002;49(1):14-8.

27. Damle SG, Gandhi M, Laheri V. Comparison of oral ketamine and oral midazolam as sedative agents in pediatric dentistry. J Indian Soc Pedod Prev Dent. 2008;26(3):97-101. doi:10.4103/0970-4388.43186

28. Pringle B, Dahlquist LM, Eskenazi A. Memory in pediatric patients undergoing conscious sedation for aversive medical procedures. Health Psychol. 2003;22(3):263-9. doi:10.1037/0278-6133.22.3.263

29. Savanheimo N, Vehkalahti MM. Five-year follow-up of children receiving comprehensive dental care under general anesthesia. BMC Oral Health. 2014;14(1):154. doi:10.1186/1472-6831-14-154

30. Peretz B, Faibis S, Ever-Hadani P, Eidelman E. Dental health behavior of children with BBTD treated using general anesthesia or sedation, and of their parents in a recall examination. ASDC J Dent Child. 2000;67(1):50-4.

31. Zaze AC, Fraga RC, Cunha RF. Evaluation of children's behavior aged 0-3 years during dental care: a longitudinal analysis. J Indian Soc Pedod Prev Dent. 2009;27(3):145-50. doi:10.4103/0970-4388.57094

32. American Academy of Pediatric Dentistry. Guideline for monitoring and management of pediatric patients during and after sedation for diagnostic and therapeutic procedures. Pediatr Dent. 2008-2009;30(7 Suppl):143-59.

33. Fewtrell MS, Kennedy K, Singhal A, Martin RM, Ness A, Hadders-Algra M, et al. How much loss to follow-up is acceptable in long-term randomised trials and prospective studies? Arch Dis Child. 2008;93(6):458-61. doi:10.1136/adc.2007.127316 